\title{
Self-Assembled Magnetically Isolated Co Nanoparticles Embedded Inside Carbon Nanotubes
}

\author{
Serghej L. Prischepa ${ }^{1,2}$, Alexander L. Danilyuk ${ }^{1}$, Andrei V. Kukharev ${ }^{1}$, \\ Francois Le Normand ${ }^{3,4}$, and Costel S. Cojocaru ${ }^{3,5}$ \\ ${ }^{1}$ Department of Micro and Nanoelectronics, Belarusian State University of Informatics and Radioelectronics, \\ 220013 Minsk, Belarus \\ ${ }^{2}$ Department of Condensed Matter Physics, National Research Nuclear University "MEPhI," 115409 Moscow, Russia \\ ${ }^{3}$ CNRS, Institut de Physique et Chimie des Matériaux de Strasbourg, University of Strasbourg, \\ 67037 Strasbourg, France \\ ${ }^{4}$ ICube/MaCEPV Laboratory, CNRS, University of Strasbourg, 67037 Strasbourg, France \\ ${ }^{5}$ Laboratoire de Physique des Interfaces et des Couches Minces, CNRS, Ecole Polytechnique, \\ Université Paris-Saclay, 91128 Palaiseau, France
}

\begin{abstract}
We investigate the influence of carbon nanotubes (CNTs) aligned array on the magnetic properties of an ensemble of densely packed Co nanoparticles (NPs) embedded inside CNTs. Samples are synthesized by chemical vapor deposition activated by current discharge plasma and hot filament. Each CNT contained only one NP of Co, which had preliminarily been formed on the surface of the $\mathrm{SiO}_{2} / \mathrm{Si}$ substrate. Co NPs are elongated along the CNT axis. The reference Co NPs ensemble and Co NPs embedded inside CNTs behave differently in a magnetic field. In the former case, Co NPs are strongly coupled by the dipole-dipole interaction (DDI); the easy axis plane is oriented parallel to the substrate. For Co-CNT samples, Co NPs are magnetically isolated. The reason for suppressing the contribution of the DDI is the magnetic anisotropy. It increases significantly because of the peculiar morphology of Co embedded in CNT and stresses. We evaluate the values of shape, magnetocrystalline, and magnetoelastic anisotropy constants. The magnetoelastic anisotropy is estimated for both the crystalline structures of Co, fcc and hcp, observed in Co NPs. The maximum stresses are reached in the case of hcp Co, when the hexagonal axis is oriented along the radial CNT direction. The influence of stresses onto the magnetic structure of $\mathrm{Co}$ inclusions is investigated by the micromagnetic simulations.
\end{abstract}

Index Terms - Carbon nanotubes (CNTs), dipole-dipole interaction (DDI), magnetic anisotropy, magnetoelasticity, nanosized cobalt.

\section{INTRODUCTION}

$\mathbf{M}$ AGNETIC structures consisting of densely packed nanosized ferromagnets represent an increased performance of devices together with a sustained interest in understanding their fundamental properties. Ferromagnetic materials with the reduced dimensions are characterized by the interplay between different magnetic anisotropy contributions. To control shape, crystalline, and elastic anisotropies, first, it is necessary to overcome the dipole interaction between nanosized magnets [1]. Second, impact on the magnetic properties of the fabrication parameters can be decisive, exaggerating the contribution of one or another anisotropy to the overall properties of the sample [2]. Conventional idea to develop nanosized magnets is based on the electrochemical deposition in porous templates. However, the nanowires fabricated by electrochemical deposition do not meet the requirements of densely packed and magnetically isolated objects. The dipole-dipole interaction (DDI) usually dominates for the density of nanosized ferromagnets greater than $n_{\mathrm{NP}} \approx 10^{9}-10^{10} \mathrm{~cm}^{-2}$ [3]. Therefore, to create a system of densely packed magnetically isolated ferromagnetic nanoparticles (NPs) with $n_{\mathrm{NP}}>10^{10} \mathrm{~cm}^{-2}$, it is necessary

Manuscript received June 4, 2018; revised July 8, 2018 and July 27, 2018 ; accepted August 3, 2018. Corresponding author: S. L. Prischepa (e-mail: prischepa@bsuir.by).

Color versions of one or more of the figures in this paper are available online at http://ieeexplore.ieee.org.

Digital Object Identifier 10.1109/TMAG.2018.2864692 to increase the contribution of the magnetic anisotropy in the total energy of the system.

To study the interplay of various magnetic anisotropy contributions in the array of densely packed nanosized Co in this paper, we fabricate samples applying a different bottom-up technique based on the chemical vapor deposition activated by current discharge plasma and hot filament (dc HF CVD) [4]. This approach allows fabricating densely packed $\left(n_{\mathrm{NP}}>10^{10} \mathrm{~cm}^{-2}\right)$ ferromagnetic NPs embedded inside vertically aligned carbon nanotubes (CNTs) [5], [6]. Thus, the problem of agglomeration of NPs is prevented. Moreover, the combination of these two types of materials (CNTs and NPs) significantly modifies the magnetic properties of the sample and increases the resistance of NPs toward their oxidation [7], [8]. Such type of CNT-based magnetic nanocomposite is a promising material for magnetoelectronics.

\section{SAMPlE FABRiCATION AND ChaRACTERIZATION}

A $\mathrm{SiO}_{2}$ layer of $8 \mathrm{~nm}$ thickness was first deposited onto the $\mathrm{Si}(100)$ substrate. Then, Co film of $5 \mathrm{~nm}$ thickness was deposited with an evaporation cell in an ultra high vacuum (UHV) chamber directly connected to the CVD reactor. Afterwards, the substrate was transferred into the UHV CVD reactor, and the metal reduction and the formation of an array of Co NPs were performed by heating up to $973 \mathrm{~K}$ at a heat rate of $10 \mathrm{~K} / \mathrm{min}$ under UHV followed by a final treatment at $973 \mathrm{~K}$ in a hydrogen/ammonia mixture at $15 \mathrm{mbar}$. These conditions were chosen to optimize the formation of the 

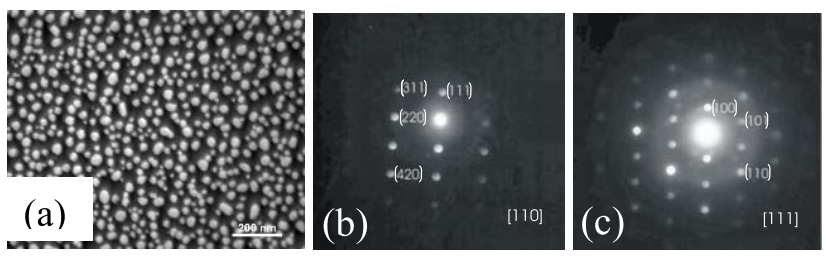

Fig. 1. SEM image of an ensemble of Co NPs on the $\mathrm{SiO}_{2} / \mathrm{Si}$ substrate (a) Patterns of TEM electron diffraction revealing (b) fcc and (c) hcp Co lattices.

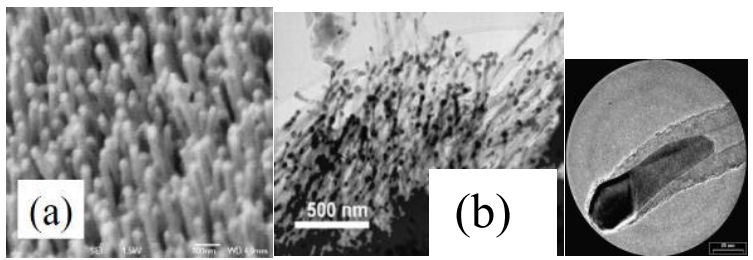

Fig. 2. (a) SEM image of vertically aligned array of CNTs on the $\mathrm{SiO}_{2} / \mathrm{Si}$ substrate. (b) TEM of the Co NPs embedded inside CNTs. Inset: typical structural shape of Co NP inside CNT obtained by TEM.

homogeneous distribution of NPs. Finally, the metal reduction and the formation of an array of Co NPs were performed in a thermally activated mixture of hydrogen and ammonia at $973 \mathrm{~K}$ during $15 \mathrm{~min}$. As a result, a reference sample consisting of an ensemble of Co NPs with an average diameter of $15 \pm 5 \mathrm{~nm}$ and $n_{\mathrm{NP}} \approx 1.2 \times 10^{10} \mathrm{~cm}^{-2}$ was synthesized on the surface of the $\mathrm{SiO}_{2} / \mathrm{Si}$ substrate. The characteristic view of an array of Co NPs is shown in Fig. 1(a). Co was observed in two modifications of the crystalline lattice, fcc and hcp, which was proven by TEM electron diffraction [Fig. 1(b) and (c), respectively].

The process of dc HF CVD was done in the mixture of $\mathrm{C}_{2} \mathrm{H}_{2}: \mathrm{H}_{2}=20: 80$ with a flow rate of $100 \mathrm{sccm}$ at $15 \mathrm{mbar}$ and a filament power of $150 \mathrm{~W}$. The process time was $6 \mathrm{~min}$ to minimize the defectiveness of CNTs and their bending. Co NPs act as a catalyst for the CNTs' growth. The results of scanning and transmission electron microscopy strongly suggest the growth of an array of vertically oriented CNTs [Fig. 2(a)] with only one Co NP inserted and located at the top of each CNT [Fig. 2(b)]. This is expected when using a plasma-activated process [4], [9].

We emphasize that most of these Co NPs are elongated, as can be seen in Fig. 2(b), with the long axis oriented parallel to the nanotube axis. The diameter of these inclusions is restricted by the inner CNT channel $(\approx 15-20 \mathrm{~nm})$, and their aspect ratio is about 5, i.e., the NPs have a cylindrical-like shape. Such resulting Co morphology embedded on top of CNT underlines the importance of diffusion processes in/on the NP bulk/surface during the CNT growth. The TEM analysis revealed the regular structure of CNTs and their low defectiveness [4] in agreement with the Raman spectroscopy [10]. The CNTs are multiwalled but with a clear and regular hollow inside. More details about the processes of dc HF CVD for the CNTs' growth and Co-CNT samples characterization can be found elsewhere [4], [9].
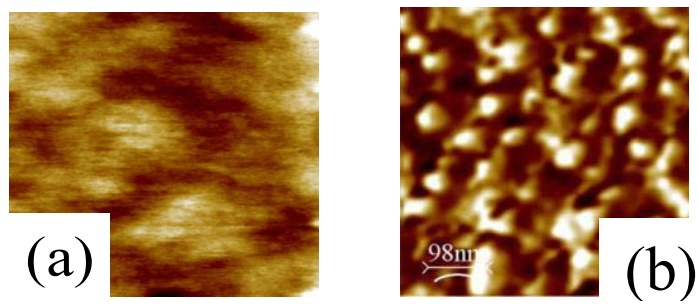

Fig. 3. (a) MFM image of Co NPs on the $\mathrm{SiO}_{2} / \mathrm{Si}$ substrate. The size of image is $1.5 \mu \mathrm{m} \times 1.5 \mu \mathrm{m}$. (b) MFM image of Co NPs embedded inside CNT.
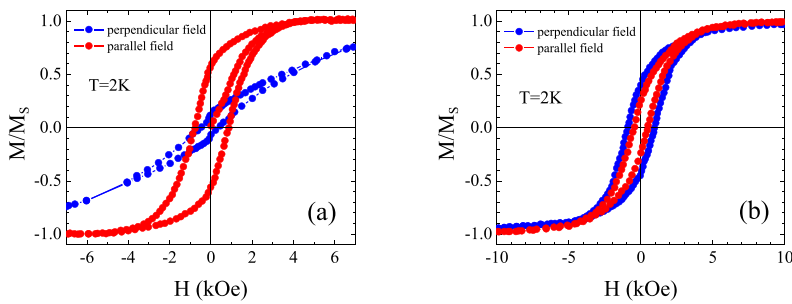

Fig. 4. Normalized magnetization $M / M_{S}$ versus magnetic field $H$ for the parallel (red) and perpendicular (blue) magnetic field orientations. $T=2 \mathrm{~K}$. (a) Reference sample. (b) Co CNT.

\section{Magnetic Properties}

Fig. 3(a) shows the magnetic force microscopy (MFM) image for the reference sample, and Fig. 3(b) shows the MFM result for an array of CNTs with Co on the top of each CNT. The intensity variations are a measurement of the attractive (repulsive) magnetic force gradient.

In the first case, it is possible to distinguish magnetic domains with an average size of about $500 \mathrm{~nm}$ encompassing a set of many NPs, which indicates strong DDI between them. For Co-CNT sample, which was grown from a reference one, the average size of a magnetic dipole is about $50 \mathrm{~nm}$, which is close to the average size of Co at the top of the CNT. Thus, Co NPs are magnetically separated from each other.

The difference in the magnetic interaction between the particles on the substrate and inside the CNT is also manifested in the shape of hysteresis loops. In Fig. 4(a), the $M(H)$ curves for the reference sample are shown for magnetic field oriented both parallel and perpendicular to the substrate. Rapid saturation in the parallel field along with greater values of the remanence and coercivity can be reasonably attributed to an axis of easy magnetization oriented parallel to the substrate. This can be naturally explained by the strong DDI between NPs, because of which the array of NPs can be considered as a thin film.

For Co-CNT sample, $M(H)$ loops approach each other for parallel and perpendicular field directions, see Fig. 4(b). Usually for an ensemble of Co NPs with the aspect ratio $\geq 3-5$, the easy axis is oriented along the cylinder, and $M(H)$ shapes for the easy and hard axes directions differ significantly [11]. Therefore, most likely, in our Co-CNT samples, there is a complex case where magnetocrystalline and, possibly, magnetoelastic anisotropy of NPs have a dispersion relative to some direction at a certain angle to the axis of the nanocylinders. This fact leads to uncertainty in the direction 
of the axis of easy magnetization, which requires additional analysis.

A better understanding of the observed changes in the magnetic behavior of Co NPs can only be achieved by analyzing the effects of different anisotropy contributions to the total anisotropy of the system and comparing them with the strength of the DDI.

\section{Discussion}

The effective magnetic anisotropy constant $K$ which includes contributions of the magnetocrystalline $\left(K_{\mathrm{MCA}}\right)$ and shape anisotropies $\left(K_{\mathrm{SA}}\right)$, the magnetoelastic contribution $\left(K_{\mathrm{MEA}}\right)$ and the DDI $\left(K_{\mathrm{DD}}\right)$, can be evaluated within the random anisotropy model, which has been successfully applied in the past to explain the properties of ferromagnetic NPs in CNT [12]

$$
K=H_{c} A^{3} M_{S} /\left[114\left(k_{B} T_{B}\right)^{2}\right] .
$$

In (1), $A$ is the exchange constant, $M_{S}$ is the saturation magnetization, and $T_{B}$ is the blocking temperature.

The results will be presented for the parallel field as follows. For the perpendicular field, the data are similar. $T_{B}(400 \mathrm{~K}), H_{C}\left(6.53 \times 10^{4} \mathrm{~A} / \mathrm{m}\right.$ for the reference sample and $3.76 \times 10^{4} \mathrm{~A} / \mathrm{m}$ for Co CNT), and $M_{S}\left(1.4 \times 10^{6} \mathrm{~A} / \mathrm{m}\right)$ were measured. For the nanosized $\mathrm{Co}$, we took from the literature $A=1.54 \times 10^{-11} \mathrm{~J} / \mathrm{m}$ [13]. Thus, for the reference sample, $K=3.77 \times 10^{5} \mathrm{~J} / \mathrm{m}^{3}$ and for Co-CNT sample, $K=2.65 \times 10^{5} \mathrm{~J} / \mathrm{m}^{3}$.

The contribution of the DDI is expressed as [14]

$$
K_{\mathrm{DD}}=(1 / 8 \pi)\left(\mu_{0} M_{S}^{2} V / \zeta^{3}\right) s_{d}
$$

where $\zeta$ is an average interparticle distance, $s_{d}=6-8$ is a lattice sum that depends on the type of lattice in the NPs arrangement and the dimensionality of the sample, and $V$ is the NP volume. From (2), we estimate $K_{\mathrm{DD}}=1.4 \times 10^{5} \mathrm{~J} / \mathrm{m}^{3}$ for the reference sample and $K_{\mathrm{DD}}=1.1 \times 10^{5} \mathrm{~J} / \mathrm{m}^{3}$ for the Co CNT. The contribution of the SA for the reference sample can be neglected due to the almost equal transverse and longitudinal demagnetizing factors. In addition, we believe that the contribution of the magnetoelastic anisotropy will be unimportant because the Co NPs are in a practically free state.

Therefore, the anisotropy constant $K$ for the reference sample is a function of only $K_{\mathrm{DD}}$ and $K_{\mathrm{MCA}}$. This results in $K_{\mathrm{MCA}}=2.4 \times 10^{5} \mathrm{~J} / \mathrm{m}^{3}$. This value is assumed to be the same for both the types of samples. The obtained $K_{\text {MCA }}$ is slightly greater than $K_{\mathrm{DD}}$. Nevertheless, the impact of the DDI in the presence of only of MCA is determined by the dipolar coupling constant $\alpha=K_{\mathrm{DD}} / K_{\mathrm{MCA}}$ [15]. There is a crucial parameter $\alpha_{\mathrm{cr}}$ that determines the transition from a single particle to the collective behavior. The reported values for fcc or hcp Co NPs are $\alpha_{\mathrm{cr}}=(0.2-0.4)(d / \zeta)^{3}$ [16]. If $\alpha>\alpha_{\mathrm{cr}}$, then DDI dominates; otherwise the MCA prevails. For our ensemble of Co NPs, we get $\alpha_{\mathrm{cr}} \approx 0.14<\alpha=0.58$, which indicates that the energy contribution of the DDI is sufficient to form regions covering many NPs, that is indeed observed experimentally.

For the Co-CNT sample, the contribution of the SA and MEA is important. Anisotropy constants $K_{\mathrm{SA}}$ and $K_{\mathrm{DD}}$ of the system of magnetic nanocylinders can be merged in a single contribution as [17]

$$
K_{\mathrm{SA}}+K_{\mathrm{DD}}=-(1 / 4) \mu_{0} M_{S}^{2}(1-3 P) .
$$

Here, $P=\left(\pi r^{2} S\right)$ is the porosity of Co nanowires assembly, $P=0.06$. From (3), we get $K_{\mathrm{SA}}+K_{\mathrm{DD}}=-5.1 \times 10^{5} \mathrm{~J} / \mathrm{m}^{3}$, which leads to $K_{\mathrm{SA}}=-6.2 \times 10^{5} \mathrm{~J} / \mathrm{m}^{3}$. Finally, on the base of the above estimations, we calculate the value of the magnetoelastic anisotropy constant $K_{\mathrm{MEA}}=5.4 \times 10^{5} \mathrm{~J} / \mathrm{m}^{3}$. This energy density is greater than those reported in [1] for Co nanowires embedded in the porous matrix. Therefore, it would be useful to estimate stresses which correspond to the obtained magnetoelasticity.

The type of Co crystalline lattice affects significantly the interpretation of data on the magnetoelastic anisotropy. For fcc Co, the $K_{\text {MEA }}$ can be evaluated as

$$
K_{\mathrm{MEA}}^{\mathrm{fcc}} \approx-(3 / 2) \lambda \sigma
$$

where $\lambda$ is the magnetostriction constant and $\sigma$ is the elastic stress. Using for fcc Co $\lambda=-50 \times 10^{-6}$ [18] and applying the obtained $K_{\text {MEA }}$ value, we estimate $\sigma \approx 7.13 \mathrm{GPa}$.

For hcp Co when the hexagonal axis is parallel to the CNT axis, $K_{\text {MEA }}$ is expressed as [19]

$$
K_{\mathrm{MEA}}^{\mathrm{hcp}}=\sigma\left(\lambda_{A}+\lambda_{B}\right) \varepsilon_{1}
$$

where $\lambda_{A}$ and $\lambda_{B}$ are the magnetostriction constants and $\varepsilon_{1}$ is the strain. From (5), we get the tensile stress $\sigma \approx 3.4 \mathrm{GPa}$.

For the hexagonal axis oriented perpendicular to the CNT axis, the situation changes significantly. The expression for $K_{\text {MEA }}^{\text {hcp }}$ depends on the orientation of the crystal magnetization $\vec{m}$ [18]. However, the exact values of direction cosines $\alpha_{i}$ of $\vec{m}$ with respect to the hexagonal axis are not known in our case. Therefore, for random $\alpha_{i}$

$$
K_{\mathrm{MEA}}^{\mathrm{hcp}}(\vec{m}) \approx B_{2} \varepsilon_{3}+B_{3}\left(\varepsilon_{1}+\varepsilon_{2}\right)
$$

where $B_{i}$ are the magnetoelastic coupling coefficients, the indices number the axes of the hexagonal crystal (index 3 corresponds to the hexagonal $c$-axis, and indices 1 and 2 correspond to the $a$ - and $b$-axes, respectively). We used $B_{2}=$ $-29 \times 10^{6} \mathrm{~J} / \mathrm{m}^{3}$ and $B_{3}=28.2 \times 10^{6} \mathrm{~J} / \mathrm{m}^{3}$ [18]. Strain $\varepsilon$ is found from the system of equations

$$
c_{i j} \varepsilon_{j}=\sigma_{i}
$$

where $c_{i j}$ are the elastic stiffness constants $\sigma_{1}=\sigma_{3}=\sigma$ and $\sigma_{2}=0$. For the considered case, we evaluate $\sigma=-9.5 \mathrm{GPa}$. Such internal elastic stresses cause the strain of the CNTs' lattice not more than $\varepsilon \approx 10^{-3}$, which is in good agreement with [20].

The performed evaluations show that the DDI is suppressed mainly by the SA and MEA. The largest contribution of the magnetoelastic anisotropy occurs when the hexagonal axis is oriented parallel to the substrate. This is confirmed by the results of the micromagnetic simulation, which was performed for hcp Co nanocylinders with diameter $20 \mathrm{~nm}$ and length $100 \mathrm{~nm}$. We applied the Nmag package [21] based on the Landau-Lifshitz-Gilbert equation. 

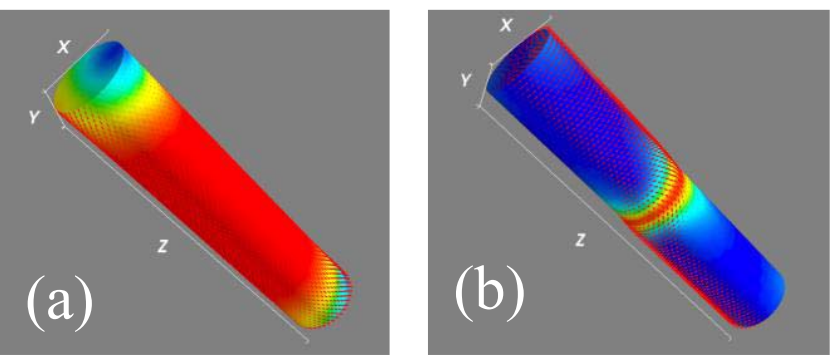

Fig. 5. Relaxed magnetization configuration of hcp Co nanocylinder of a diameter of $20 \mathrm{~nm}$ and a length of $100 \mathrm{~nm}$. (a) $K_{\mathrm{MEA}}=0$. (b) $K_{\mathrm{MEA}}=$ $5.35 \times 105 \mathrm{~J} / \mathrm{m}^{3}$. Red (blue)—magnetization direction parallel (perpendicular) to the nanocylindrical axis.

The $z$-axis is oriented along the nanocylindrical axis (i.e., the CNT orientation), and the $x y$ plane is lying in the radial direction. The $x$-axis coincides with the hexagonal axis of the hcp Co. The simulation shows that under such conditions, the relaxed magnetization configuration of the cylinder depends on $K_{\mathrm{MEA}}$, see Fig. 5. For $K_{\mathrm{MEA}}=0$, the magnetization of the Co nanocylinder is homogeneous and oriented along the $z$-axis, see Fig. 5(a). The presence of the magnetoelastic stresses, $K_{\text {MEA }}=5.35 \times 10^{5} \mathrm{~J} / \mathrm{m}^{3}$, breaks nanocylinder in two domains, see Fig. 5(b).

The performed simulations of the magnetic structure of Co nanocylinders should be considered only as evaluation results showing that with the magnetoelastic component nanocylinders may be partitioned into domains, which leads to a decrease in remanence and coercivity and to isotropy of the magnetic properties. This is in good agreement with the experimental data.

\section{CONCLUSion}

We have demonstrated experimentally the impact of CNTs on the magnetic properties of the ensemble of densely packed Co NPs. We have shown that both SA and MEA are crucial in suppressing the DDI. High magnetoelasticity is caused mainly by the original self-assembled bottom-up fabrication process. The magnetoelastic anisotropy has been evaluated for both the observed crystalline structures of Co, fcc and hcp. The noticeable magnetoelasticity is due to the presence of the hcp crystalline phase. Such an approach in the fabrication of an ensemble of anisotropic NPs can be very useful in the design of magnetoelectronic devices with high packing density.

\section{REFERENCES}

[1] J. Sánchez-Barriga et al., "Interplay between the magnetic anisotropy contributions of cobalt nanowires," Phys. Rev. B, Condens. Matter, vol. 80 , no. 18 , p. 184424 , Nov. 2009.
[2] M. Gong, Q. Dai, and S. Ren, "Magnetic dipolar interaction induced cobalt nanowires," Nanotechnology, vol. 27, p. 07LT02, Jan. 2016.

[3] M. Vázquez, K. Pirota, J. Torrejón, D. Navas, and M. Hernández-Vélez, "Magnetic behaviour of densely packed hexagonal arrays of Ni nanowires: Influence of geometric characteristics," J. Magn. Magn. Mater, vol. 294, no. 2, pp. 174-181, 2005.

[4] C. S. Cojocaru and F. Le Normand, "On the role of activation mode in the plasma- and hot filaments-enhanced catalytic chemical vapour deposition of vertically aligned carbon nanotubes," Thin Solid Films, vol. 515 , pp. 53-58, Sep. 2006.

[5] A. L. Danilyuk et al., "Interplay between exchange interaction and magnetic anisotropy for iron based nanoparticles in aligned carbon nanotube arrays," Carbon, vol. 68, pp. 337-345, Mar. 2014.

[6] A. L. Danilyuk et al., "Impact of CNT medium on the interaction between ferromagnetic nanoparticles," Europhys. Lett., vol. 117, no. 2, p. $27007,2017$.

[7] W. Baaziz et al., "High-density monodispersed cobalt nanoparticles filled into multiwalled carbon nanotubes," Chem. Mater, vol. 24, pp. 1549-1551, Apr. 2012.

[8] A. S. Andreev et al., "Magnetic and dielectric properties of carbon nanotubes with embedded cobalt nanoparticles," Carbon, vol. 114, pp. 39-49, Apr. 2017.

[9] J. M. Mane, C. S. Cojocaru, A. Barbier, J. P. Deville, B. Thiodjio Sendja, and F. Le Normand, "GISAXS study of the alignment of oriented carbon nanotubes grown on plain $\mathrm{SiO}_{2} / \mathrm{Si}(100)$ substrates by a catalytically enhanced CVD process," Phys. Status Solidi A, vol. 204, pp. 4209-4229, Dec. 2007.

[10] A. L. Danilyuk, A. V. Kukharev, C. S. Cojocaru, F. Le Normand, and S. L. Prischepa, "Impact of aligned carbon nanotube array on the magnetic properties of ferromagnetic nanocylinders," to be publised, doi: 10.1016/j.carbon.2018.08.024.

[11] C. Li et al., "Manipulation of morphology and magnetic properties in cobalt nanowires," AIP Adv., vol. 7, no. 5, p. 056229, 2017.

[12] A. L. Danilyuk et al., "Manifestation of coherent magnetic anisotropy in a carbon nanotube matrix with low ferromagnetic nanoparticle content," New J. Phys., vol. 17, p. 023073, Feb. 2015.

[13] E. Girt et al., "A method for measuring exchange stiffness in ferromagnetic films," J. Appl. Phys., vol. 109, no. 7, p. 07B765, 2011.

[14] M. A. Załuska-Kotur and M. Cieplak, "Dipole interactions with random anisotropy: A local-mean-field study," Europhys. Lett., vol. 23, pp. 85-90, Jul. 1993.

[15] V. Russier, C. Petit, J. Legrand, and M. P. Pileni, "Collective magnetic properties of cobalt nanocrystals self-assembled in a hexagonal network: Theoretical model supported by experiments," Phys. Rev. B, Condens. Matter, vol. 62, pp. 3910-3916, Aug. 2000.

[16] D. Kechrakos and K. N. Trohidou, "Dipolar interaction effects in the magnetic and magnetotransport properties of ordered nanoparticle arrays," J. Nanosci. Nanotechnol., vol. 8, pp. 2929-2943, Jun. 2008.

[17] F. Vidal et al., "Mechanism of localization of the magnetization reversal in $3 \mathrm{~nm}$ wide Co nanowires," Phys. Rev. Lett., vol. 109, p. 117205, Sep. 2012.

[18] D. Sander, "The correlation between mechanical stress and magnetic anisotropy in ultrathin films," Rep. Prog. Phys., vol. 62, pp. 809-858, May 1999.

[19] C. H. Lee, H. He, F. J. Lamelas, W. Vavra, C. Uher, and R. Clarke, "Magnetic anisotropy in epitaxial Co superlattices," Phys. Rev. B, Condens. Matter, vol. 42, pp. 1066-1069, Jul. 1990.

[20] M.-F. Yu, O. Lourie, M. J. Dyer, K. Moloni, T. F. Kelly, and R. S. Ruoff, "Strength and breaking mechanism of multiwalled carbon nanotubes under tensile load," Science, vol. 287, no. 5453, pp. 637-640, Jan. 2000.

[21] Computational Micromagnetism. Accessed: May 18, 2018. [Online]. Available: http://nmag.soton.ac.uk/nmag/ 